\title{
Electron energy-loss spectroscopy for direct visualization of gas adsorption sites
}

Wei-Chang Yang ${ }^{1}$, Canhui Wang ${ }^{2}$ and Renu Sharma ${ }^{3}$

${ }^{1}$ National Insitute of Standards and Technology, United States, ${ }^{2}$ University of Maryland, Gaithersburg, Maryland, United States, ${ }^{3}$ National Institute of Standards and Technology, United States

Over the last two decades, environmental scanning-transmission electron microscope (ESTEM) has become a method of choice for measuring and understanding atomic scale mechanisms of heterogenous catalytic reactions. The adsorption of reactant molecules on selective metal catalyst surfaces is the first step of fundamental importance at it indicates catalytically active sites. Although the gas molecules do not cause strong electron scattering to generate enough contrast for imaging, surface modulation of the $\mathrm{Au}$ nanoparticles due to $\mathrm{CO}$ adsorption has been reported an indirect method to identify gas adsorption sites [1]. Recently, water adsorption on reconstructed $\mathrm{TiO}_{2}$ surfaces has also been reported [2]. Here, we show an alternative method using the core-loss electron energy-loss maps to directly visualize the gas adsorption locations.

We employ an ESTEM equipped with a monochromated high brightness field-emission gun (XFEG) with $0.08 \mathrm{eV}$ energy resolution, dual-EELS, operated at $80 \mathrm{keV}$ for acquiring STEM-EELS maps. Spectrum images acquired in gaseous environments are a three-dimensional data cube that includes energy-loss information in the $\mathrm{Z}$ direction at each pixel of a STEM-ADF image. Environmental contribution of the gaseous component was extracted from a square area where only relevant gas is present in the original STEM-EELS map. The rescaled image is subtracted from the original STEM-EELS map to obtain the adsorption of the gas molecules. Background subtraction method depends on the complexity of the energy-loss region. For example, a multiple linear least square fit using reference spectra was employed for the $\mathrm{H} \mathrm{K}$-edge while a power-law fit was used for $\mathrm{C}$ k-edge.

In order to discern the active sites for a room temperature $\mathrm{CO}$ disproportionation reaction by $\mathrm{Au}$ localized surface plasmon resonance (LSPR), we have successfully applied this procedure to identify the location of $\mathrm{CO}$ adsorption on shape-controlled Au nanoprisms (Figure 1a-c) in various gas pressures using C K-edge maps [3]. Interestingly, the C K-edge intensities (marked green) were located on the edges, where two crystallographic planes meet, instead of the surface as revealed by a series of images at varying tilted angles (Figure 1a-c). Furthermore, we used the same method to obtain $\mathrm{H}_{2}$ adsorption maps on $\mathrm{Al}$ nanoparticles (Figure $1 \mathrm{~d}-\mathrm{f}$ ), surrounded by a shell of $\mathrm{Al}_{2} \mathrm{O}_{3}$, to probe $\mathrm{H}_{2}$ dissociations assisted by $\mathrm{Al}$ LSPR, which have been reported at room temperature in the literature [4]. The presence of H K-edge (13 $\mathrm{eV}$, Figure 1e, red line) in the low-loss region complicates the extraction of its contribution as the Al bulk plasmon peak and other spectral features associate with $\mathrm{Al}_{2} \mathrm{O}_{3}$ are also present in the same region. Therefore, the multiple linear least square fitting was used to extract the $\mathrm{H}_{2}$ adsorption map (Figure 1f) where $\mathrm{H}_{2}$ adsorption was found to be limited to the amorphous $\mathrm{Al}_{2} \mathrm{O}_{3}$ shell surround the $\mathrm{Al}$ core. A detailed discussion of the procedure will be further evaluated using other examples, such as $\mathrm{CO}_{2}$ adsorption on graphite, oxygen adsorption of boron nitride nanotubes. 


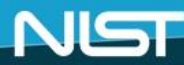
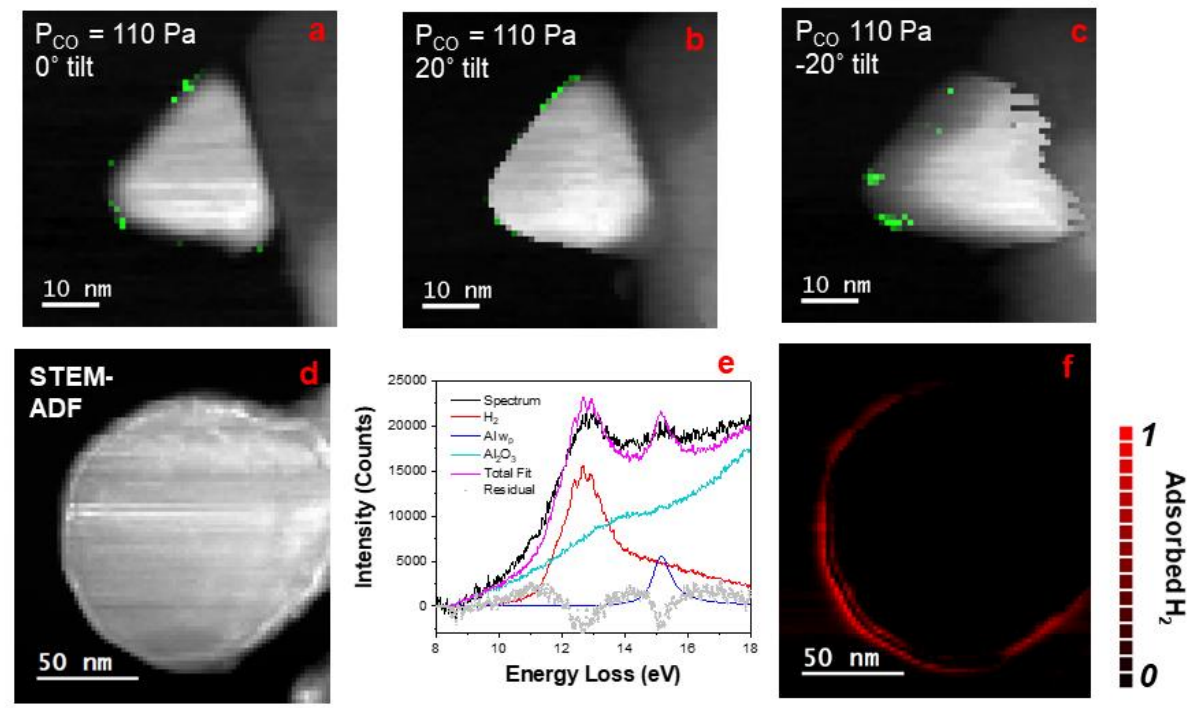

Figure 1. Figure 1 (a-c) Carbon maps (green), acquired by tilting the particle by $\pm 20^{\circ}$ and after removing the environmental contribution of $\mathrm{CO}$ from the images, showing the $\mathrm{CO}$ absorption on specific edges of an Au nanoprism (see reference 2 for details). (d) An ADF image of an Al nanoparticle in $100 \mathrm{~Pa}$ of $\mathrm{H} 2$ (e) EELS spectra showing the contributions from H2, Al (metal), A12O3 obtained after multiple linear least square fitting, (f) $\mathrm{H} 2$ map showing the $\mathrm{H} 2$ adsorption is confined to the $\mathrm{A} 12 \mathrm{O} 3$ shell.

\section{References}

[1] H. Yoshida et al., Science 335 (2012) 317.

[2] W. Yuan et al., Science 367 (2020) 428.

[3] W-C. D. Yang, Nature Materials 18 (2019), 614.

[4] S. Mukherjee, S. et al., J. American Chemical Society 136, (2014) 64. 